\title{
An Energy Harvesting Nonvolatile Sensor Node and Its Application to Distributed Moving Object Detection
}

\author{
Yongpan Liu, Yiqun Wang, Hongyang Jia, Shan Su, Jinghuan Wen, Wenzhu Zhang, \\ Lin Zhang and Huazhong Yang \\ Dept. of Electronic Engineering, Tsinghua University \\ Tsinghua National Laboratory for Information Science and Technology \\ Beijing, 100084, P.R.China \\ ypliu@tsinghua.edu.cn, yanghz@tsinghua.edu.cn
}

\begin{abstract}
Energy harvesting sensor nodes based on real nonvolatile processors are demonstrated to show the desirable characteristics of those systems, such as no battery, zero standby power, microsecond-scale sleep and wake-up time, high resilience to random power failures and fine-grained power management. Furthermore, we show its applications to a distributed moving object detection system, one of novel nonvolatile computing systems.
\end{abstract}

\section{Categories and Subject Descriptors}

C.3 [Special-Purpose and Application-Based Systems]: Microprocessor/microcomputer applications

\section{Keywords}

Nonvolatile Sensor Node, Object Detection

\section{INTRODUCTION}

Energy harvesting techniques [1] provide a possible way to eliminate the need for a battery from wireless sensor nodes. However, power variations and intermittent availability patterns from the energy harvesting module make the traditional sensor nodes unreliable and even fail. This limitation comes from the restriction of volatile processors. Given a volatile processor based sensor node, the system state will become lost when powered off. Though it may be possible to store the system state to the off-chip nonvolatile memory, it would be both time and energy consuming. As a result, such back-up scheme is not fast enough and inefficient to follow the power intervals in a real energy harvesting module. In many applications, it is desirable for the processor to remember its state quickly and efficiently before powered off. When powered on, the processor can resume computing and sensing at the exact place before the power failure. In this way, the sensor node can work effectively in an energy harvesting environment.

Next, we will demonstrate the prototype of an energydriven sensor node based on a nonvolatile processor. The proposed node consists of an energy harvesting module, a nonvolatile computing and sensing module and a power efficient wireless transceiver. It addresses the above challenges

Copyright is held by the author/owner(s). IPSN'12, April 16-20, 2012, Beijing, China. ACM 978-1-4503-1227-1/12/04. facing the self-power sensor node based on the volatile processor. Based on the ferroelectric nonvolatile memory technologies, the nonvolatile processor [2] based sensor node has the following advantages: I) zero-standby power, II) instant on and off, III) high resilience to power failures, IV) finegrained power management supported. To show the system's high resilience to power intervals and its continuous operation in an energy driven mode, a demo is built where the sensor node works continuously under a $1 \mathrm{Khz}$ square wave power supply. The setup is depicted in Fig. 1. Here, the sensor node is running a counter program. After each power failure, the system restarts from the value before the nearest power break point, instead of the initial system state. This system is one of our demonstration platforms.

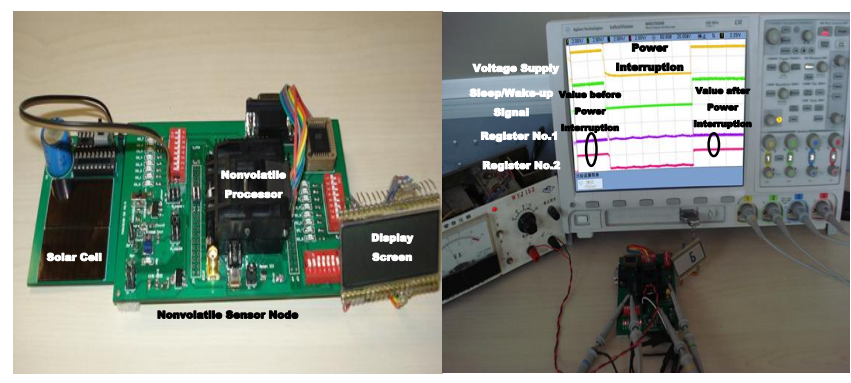

Figure 1: Proposed Energy Driven Nonvolatile Sensor Node

Furthermore, we will demonstrate a moving objects detection system based on the proposed energy-driven nonvolatile sensor node. This system is depicted in Fig. 2. Each nonvolatile sensor node is equipped with a solar cell energy harvester with no batteries. The energy source can be the sun outdoors or light source indoors. Given an energy source, the sensor nodes continuously count the number. When a moving object (e.g., a person) comes in between the light and the sensor node, power to the sensor node is cut off. The nonvolatile sensor node will remember the current state and wait for the moving object passes by. After that, the power supply is recovered, and the sensor node will continue to count. The counting number and related information can be stored in the local nonvolatile memory or wirelessly transferred to the remote data center. An object recognition algorithm is used in the data center to analyze the object occurring time 
and other information. A graphic user interface (GUI) is provided to show the real-time detecting results.

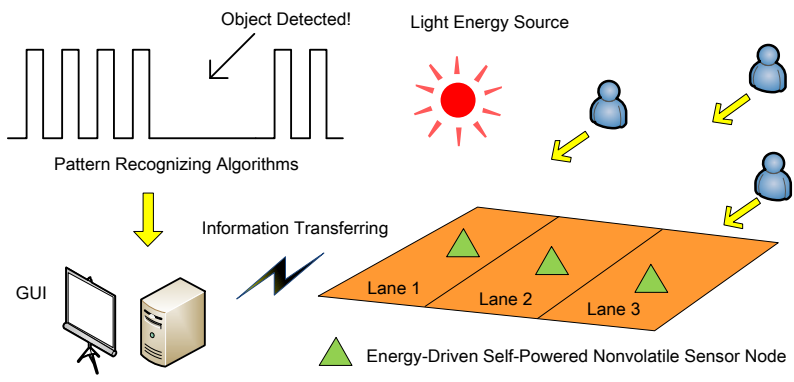

Figure 2: The Proposed Moving Objects Detection System

\section{ON-SITE DEMONSTRATION}

The on-site demonstration will contain two parts. The first one provides some stand alone energy driven sensor nodes for volunteers. Those sensor nodes will powered by the pressure, vibration or other mechanical energy harvesters. People can participate the activities and experience the new way in which nonvolatile sensor nodes work. The other interesting part shows the principle of energy driven technique in a moving objects detecting wireless network. It will accomplish the mission of counting people through the entrances. As is shown in Fig. 2, it consists of three sensor nodes representing three entrances from different directions, a light generator to simulate sunlight indoors, a data collection point and a GUI display.

We will call for volunteers in the exhibition hall, ask them to walk pass one of the three sensor nodes to block the light path from the light generator to the node. After a certain period, the collecting node will aggregate statistics from each node, calculate the number and the times that objects pass by the node and update the information on the GUI. A data collection point will work with a $2.4 \mathrm{GHz} 802.15 .4$ channel to acquire counting numbers from the sensor nodes. It can be considered as the gateway node with sufficient energy supply and strong processing capacity. We runs time synchronization and moving objects counting algorithm on it.

\section{KEY TECHNIQUES}

The most novel technique used in this demo is the nonvolatile processor based energy driven system. This system consists of the energy harvesting module (solar cell), the power management unit (PMU) and the nonvolatile processor (NVP), shown in Fig. 3. Only several square centimeters solar cell is needed to provide $6 \mathrm{~V}$ and more than $5 \mathrm{~mW}$ power supply under medium sun light which is sufficient for the processor. The PMU has functions of energy detection and voltage regulation. It measures the energy stored on the capacitor and generates activation signals to NVP as well as regulates voltage supply properly to the NVP.

The nonvolatile processor [2] is an emerging approach to nonvolatile computing. In our NVP, the registers are all replaced by the ferroelectric flip-flops. Its work mode can be changed between zero-leakage sleep mode and normal

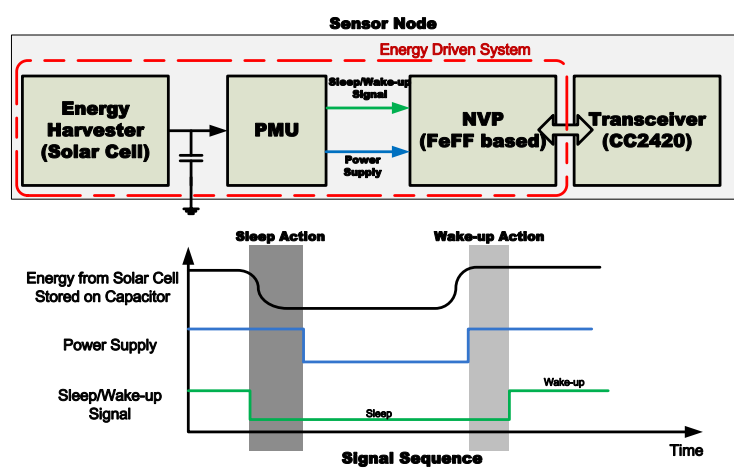

Figure 3: Architecture of Energy Driven Sensor Node and Its Controlling Signal Sequence

mode driven by intermittently available voltage supply. We designed a nonvolatile processor based on the hybrid ferroelectric CMOS process for the energy driven sensor node. We draw the transformations curves between the sleep and wake-up mode in Fig. 3. When the PMU detects a drop in the voltage level, it first generates the sleep signal and maintains the power supply via the capacitor until system state is stored in nonvolatile cells then cuts off the power. To wake up, the PMU detects power regaining, then provides power supply to the NVP until power is stable and after that generates the wake-up signal to restart the NVP. According to the measured results, the wake-up action consumes less than $100 \mu s$ and the sleep action consumes around $50 \mu s$ which reveals our system can work under a frequently interrupted power supply.

In the network level, a synchronization algorithm should be implemented among the collecting point and sensor nodes. After a certain period of time, the global time should be refreshed and synchronized with each node.

\section{CONCLUSIONS}

The energy driven nonvolatile sensor node provide an efficient way of eliminating power and lifetime constraint and the possibility of emergency detection based on energy interruption. Moreover it can continuously assemble information even in a poor power supply condition. We show above advantages in our moving object detection demo. Such NVP based systems offer tremendous potential at the interface of the cyber space and physical world.

\section{ACKNOWLEDGEMENT}

Thanks for many helpful suggestions from Prof. Xiaobo Sharon Hu. This work was supported by ROHM Co., NSFC under grant 60976032, ZX under contract 2010ZX03006-00301 and 863 under 2009AA01Z130.

\section{REFERENCES}

[1] A. Kansal, J. Hsu, S. Zahedi, and M. Srivastava, "Power management in energy harvesting sensor networks," ACM Transactions on Embedded Computing Systems (TECS), vol. 6, no. 4, p. 32, 2007.

[2] Y. Wang, Y. Liu, Y. Liu, D. Zhang, S. Li, B. Sai, M. Jiang, and H. Yang, "A compression-based area-efficient recovery architecture for nonvolatile processors," in Proceedings of DATE, 2012. 